\title{
Effect of Bacteriophages on Viability and Growth of Co-cultivated Weissella and Leuconostoc in Kimchi Fermentation
}

\author{
Se-Jin Kong and Jong-Hyun Park* \\ Department of Food Science and Biotechnology, Seongnam 13120, Gachon University, Seongnam 13120, Republic of Korea
}

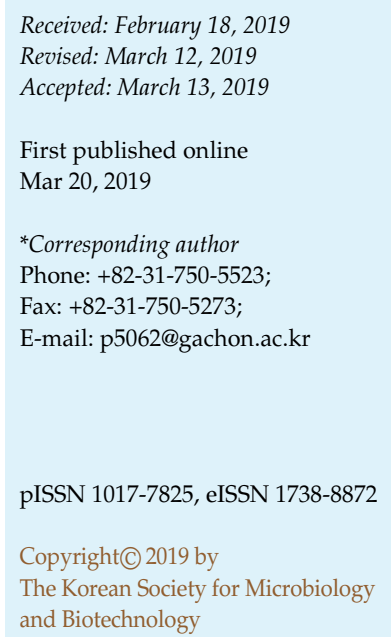

\begin{abstract}
This study aimed to understand the survival and growth patterns of bacteriophage-sensitive Weissella and Leuconostoc strains involved in kimchi fermentation. Dongchimi kimchi was prepared, and Weissella and Leuconostoc were co-cultivated in the dongchimi broth. Weissella cibaria KCTC 3807 growth was accompanied by rapid lysis with an increase in the bacteriophage quantity. Leuconostoc citreum KCCM 12030 followed the same pattern. The bacteriophage-insensitive strains W. cibaria KCTC 3499 and Leuconostoc mesenteroides KCCM 11325 survived longer under low $\mathrm{pH}$ as their growth was not accompanied by bacteriophages. The bacteriophage lysate of W. cibaria KCTC 3807 accelerated and promoted the growth of Leuconostoc. Overall, our results show that bacteriophages might affect the viability and population dynamics of lactic acid bacteria during kimchi fermentation.
\end{abstract}

Keywords: Weissella, Leuconostoc, bacteriophage, population dynamics, kimchi
Among the microorganisms involved in kimchi fermentation, lactic acid bacteria (LAB) play the most important role. LAB produce lactic acid, acetic acid, ethanol, carbonic acid gas, aldehydes, and so on, giving kimchi its unique flavor [1]. The LAB present in kimchi tend to vary such that those involved in initial and late fermentation sequentially grow and influence each other [2]. Different raw materials, ingredients, and fermentation conditions have been suggested to significantly affect LAB profiles during kimchi fermentation [3, 4]. In addition to $\mathrm{LAB}$, several types of $\mathrm{LAB}$ bacteriophages have been detected and isolated during kimchi fermentation. During cheese fermentation, the bacteriophages deter fermentation by stopping milk acidification [5]. Recently, studies on the control of food production during fermentation through the control of LAB bacteriophages have been conducted [6]. In pickle fermentation, the bacteriophages of Weissella, Leuconostoc, and Lactobacillus were detected sequentially [7]. Therefore, it was expected that bacteriophages might affect the succession of lactic acid bacteria $[8,9]$.

Bacteriophages are viruses that infect specific bacterial hosts and are known to outnumber bacteria ten-fold, estimated at over $10^{31}$ bacteriophages globally [10]. Bacteriophages exhibit two life cycles: lytic and lysogenic.
In the lysogenic life cycle, the bacteriophage is introduced into the bacterial genome as a prophage: this prophage can later be induced by environmental stresses leading to virus production and host cell lysis [11]. Such bacterial lysates have been suggested to supply energy to the marine ecosystem food web, where bacteriophages may function as nutrient recyclers [12]. Therefore, bacteriophages might also be related to LAB lysis during kimchi fermentation, resulting in $\mathrm{LAB}$ succession and nutrient recycling in the microecosystem of kimchi. As such, this study investigated the effect of bacteriophages on the viability and growth of Leuconostoc and Weissella to better understand the successive population dynamics during early kimchi fermentation.

Kimchi can be generally classified in the two types, ordinary and watery kimchi. The watery kimchi is made mainly of cabbage or radish. As a watery kimchi, dongchimi was selected as a model from many types of kimchi for easy analysis. First, Dongchimi was prepared with radishes from a local market. Radishes were cut into pieces of about $3 \mathrm{~cm}$ in height, width, and length and placed in a container. Seven percent salt water was added to the radishes at a ratio of $1: 1(\mathrm{w} / \mathrm{v})$. Then, green onions, garlic, and ginger were added to the mix at $3 \%, 1 \%$, and $0.5 \%$ $(\mathrm{w} / \mathrm{w})$ of the radishes, respectively [13]. The Dongchimi 
mixture was homogenized for $10 \mathrm{~min}$ by a stomacher (Stomacher Power Mixer, BNF Korea, Korea), centrifuged at $8,000 \times \mathrm{g}$ for $10 \mathrm{~min}$, and the supernatant was sterilized with a $0.22 \mu \mathrm{m}$ filter (Millipore, USA). The sterilized solution used as the culture broth was stored at $-80^{\circ} \mathrm{C}$. Lactic acid bacteria (LAB) used here were Weissella cibaria KCTC 3807, W. cibaria KCTC 3499, Leuconostoc citreum KCCM 12030, and L. mesenteroides subsp. mesenteroides KCCM 11325. Among them, only the bacteriophages of $W$. cibaria KCTC 3807 and L. citreum KCCM 12030 were induced during kimchi fermentation, and however, were not instead induced by mitomycin C (Sigma-Aldrich, USA).

To analyze LAB growth and bacteriophage presence during dongchimi fermentation, Weissella and Leuconostoc were inoculated separately or simultaneously into the fresh Dongchimi broth and fermentation was carried out at $7^{\circ} \mathrm{C}$ for 10 days. To differentially count Weissella and Leuconostoc in the co-culture, they were spread on MRS agar (de ManRogosa-Sharp, Oxoid, Italy) supplemented with meropenem ( $2 \mu \mathrm{g} / \mathrm{ml}$, Sigma-Aldrich), and count was determined by subtracting the number of Leuconostoc from the total number of LAB. The $\mathrm{pH}$ of the culture was also measured. To determine viability of LAB strains at different $\mathrm{pH}$, the bacterial cells were cultured in MRS broth at $9 \log \mathrm{CFU} / \mathrm{ml}$,

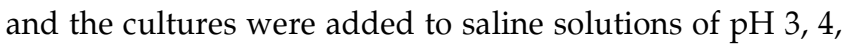
and 5 at $7 \log \mathrm{CFU} / \mathrm{ml}$. After exposure for 0,24 , and $48 \mathrm{~h}$, the live bacteria were counted by plating on MRS agar [15]. The effects of bacteriophage-sensitive $W$. cibaria on the growth of Leuconostoc spp. in Dongchimi broth was analyzed. W. cibaria KCTC 3807 was cultured in broth for 5 days until the bacteriophage count reached maximum. The cultured broth was micro-filtered to remove the pre- inoculated W. cibaria, and then Leuconostoc was cultured in the broth. Meanwhile, a Leuconostoc single culture was inoculated in fresh broth as a control. The cultures were incubated for 14 days and each bacteriophages of Weisella and Leuconostoc were quantified by the plaque assay using MRS agar.

W. cibaria KCTC 3807 (W. cibaria 3807) and L. citreum KCCM 12030 (L. citreum 12030) were inoculated simultaneously to carry out fermentation (Fig. 1A). W. cibaria 3807 reached about $5.6 \log \mathrm{CFU} / \mathrm{ml}$ at day 3 and then died off rapidly, whereas the quantity of bacteriophages increased. L. citreum 12030 grew to $6 \log \mathrm{CFU} / \mathrm{ml}$ after day 5, and then died off as the bacteriophage quantity increased. W. cibaria 3807 bacteriophages increased to about $5.6 \log \mathrm{PFU} / \mathrm{ml}$ on day 3 of fermentation, rose to $7.8 \mathrm{log} \mathrm{PFU} / \mathrm{ml}$ on day 7 , and finally decreased. L. citreum 12030 bacteriophages increased after day 3 of fermentation and then decreased to $7.5 \mathrm{log}$ $\mathrm{PFU} / \mathrm{ml}$ at day 7 . When the number of bacteriophages increased, the quantity of each host strain decreased rapidly. Therefore, these results showed that the decline in host bacteria might be lysis by bacteriophages.

W. cibaria 3807 and L. mesenteroides KCCM 11325 ( $L$. mesenteroides 11325) were inoculated simultaneously to carry out fermentation (Fig. 1B). Similarly to the above results, the number of $W$. cibaria 3807 releasing bacteriophages decreased rapidly after day 5 of fermentation when the bacteriophage count reached maximum. However, L. mesenteroides 11325, which does not release phages, appeared to maintain bacterial numbers steadily even at low $\mathrm{pH}$ until fermentation ceased, as it seemed to survive for 5 days under $\mathrm{pH} 4.0$.

W. cibaria KCTC 3499 (W. cibaria 3499) and L. citreum 12030 were inoculated simultaneously to carry out fermentation
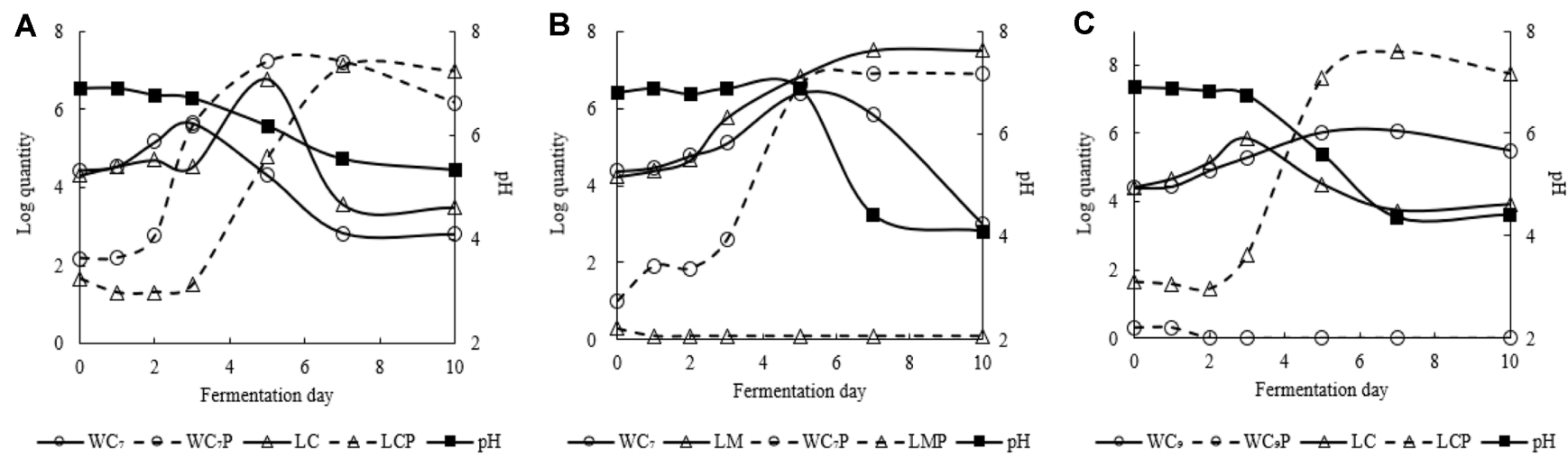

Fig. 1. Viable count profiles of W. cibaria, L. citreum, and L. mesenteroides with bacteriophages at co-cultures in Dongchimi kimchi at $7^{\circ} \mathrm{C}$. Symbols: $\mathrm{WC}_{7}$ and $\mathrm{WC}_{7} \mathrm{P}, \mathrm{W}$. cibaria KCTC 3807 and its bacteriophage; $\mathrm{WC}_{9}$ and $\mathrm{WC}_{9} \mathrm{P}, W$. cibaria $\mathrm{KCTC} 3499$ and its bacteriophage; LC and LCP, L. citreum KCCM 12030 and its bacteriophage; LM and LMP, L. mesenteroides KCCM 11325 and its bacteriophage. 
Table 1. Viability of lactic acid bacteria at $\mathrm{pH} 4.0$ and phage detection during kimchi fermentation.

\begin{tabular}{lcccc}
\hline \multirow{2}{*}{ Strains } & \multicolumn{3}{c}{ Viability at pH $4.0(\%)$} & Bacteriophage detection \\
\cline { 2 - 4 } & $0 \mathrm{~h}$ & $24 \mathrm{~h}$ & $48 \mathrm{~h}$ & at kimchi fermentation \\
\hline W. cibaria KCTC 3807 & 100.0 & 100.0 & 100.0 & + \\
W. cibaria KCTC 3499 & 100.0 & 94.7 & 89.3 & - \\
L. citreum KCCM 12030 & 100.0 & 99.9 & 96.4 & + \\
L. mesenteroides KCCM 11325 & 100.0 & 97.5 & 93.9 & - \\
\hline
\end{tabular}

Symbols: W, Weissella; L, Leuconostoc; +, bacteriophage induction; -, bacteriophage non-inducted

Viability was determined by dividing CFU of LBA at 24 or $48 \mathrm{~h}$ by CFU at $0 \mathrm{~h}$.

(Fig. 1C). W. cibaria 3499 reached about $6.1 \log \mathrm{CFU} / \mathrm{ml}$ at day 5 of fermentation and maintained this level. Contrarily, L. citreum 12030 reached about $5.8 \log \mathrm{CFU} / \mathrm{mL}$ on day 3 and then decreased rapidly. $W$. cibaria 3499 bacteriophages were not detected during fermentation. W. cibaria 3499 survived, maintaining its bacterial count until the end of fermentation. However, L. citreum 12030 bacteriophages increased to about $8.4 \log \mathrm{PFU} / \mathrm{ml}$ and then decreased after day 7. L. citreum 12030 decreased rapidly when bacteriophages were induced. Therefore, it seemed that the number of LAB decreased when phages increased $[7,8]$. The $\mathrm{pH}$ of the culture was reduced from $6.8-6.9$ to $4.1-5.3$ on the tenth day of fermentation [3]. W. cibaria 3499 seemed to survive when exposed to $\mathrm{pH} 4.0$ for 5 days. This showed that LAB viability might be related to the presence of bacteriophages rather than the $\mathrm{pH}$ during the fermentation. Additionally, all the strains survived in a $\mathrm{pH} 4$ solution with a survival rate of $89 \%$ or more following $48 \mathrm{~h}$ of exposure (Table 1 ). Therefore, low $\mathrm{pH}$ did not impact primally bacterial mortality, whereas the presence of bacteriophages did.

Finally, the effects of the bacteriophage-lysed Weisella dongchimi broth on the growth of Leuconostoc were analyzed. In the sterilized fresh Dongchimi broth, L. citreum 12030 grew to a maximum of about $7.8 \log$ CFU/ml on day 10 . Contrarily, in the Dongchimi broth containing bacteriophagelysed W. cibaria 3807, L. citreum 12030 reached a maximum of about $7.7 \log \mathrm{CFU} / \mathrm{ml}$ on day 5 (Fig. 2A), which was the fastest growth by five days. It was confirmed that L. citreum 12030 growth was promoted in Dongchimi broth containing bacteriophage-lysed $W$. cibaria 3807 . The bacteriophage lysate appeared to act as a growth activator, accelerating L. citreum 12030 growth under the given conditions. Therefore, bacteriophages might contribute to recycling nutrients for the other LAB through the lysis of one LAB. As shown in Fig. 2B, in the sterilized fresh Dongchimi broth, L. mesenteroides 11325 grew to about $7.2 \log$ CFU / ml at maximum on day 5. In contrary, in Dongchimi broth containing W. cibaria 3807 lysate, L. mesenteroides 11325
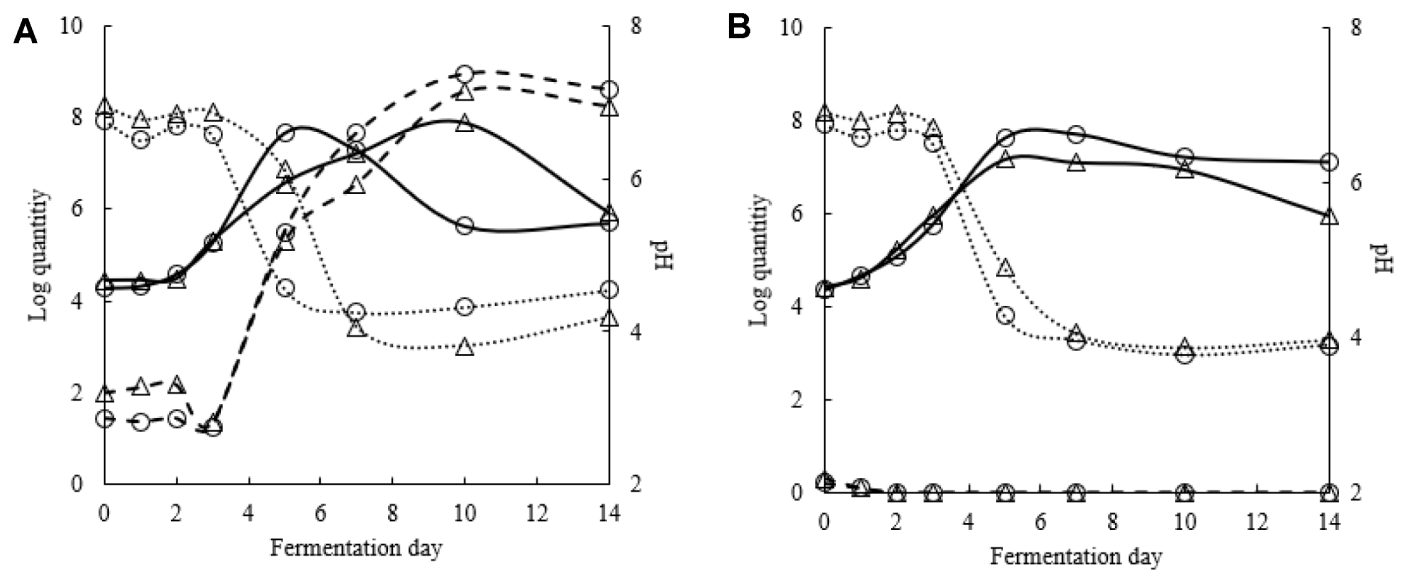

Fig. 2. Leuconostoc growth comparison in fresh Dongchimi broth $(\triangle)$ or bacteriophage-lyzate Dongchimi broth $(\bigcirc)$ at $7^{\circ} \mathrm{C}$ for L. citreum KCCM 12030 (A) and L. mesenteroides KCCM 11325 (B).

The bacteriophage-lyzate broth was prepared after microfiltration of pre-cultured Dongchimi by bacteriophage-sensitive W. cibaria KCTC 3807 to remove the live bacteria. The dashed and dotted lines indicate Leuconostoc phage quantities and $\mathrm{pH}$ values, respectively. 
reached its highest bacterial count of $7.6 \log \mathrm{CFU} / \mathrm{ml}$ on day 5, greater than the culture in the fresh Dongchimi broth. In the Dongchimi broth with W. cibaria 3807 lysate, L. mesenteroides 11325 growth was promoted and mortality was reduced.

In summary, bacteriophage-sensitive LAB survival was short as they died off due to bacteriophage induction or propagation [3, 7]. Contrarily, bacteriophage-insensitive LAB survived relatively longer, even at low $\mathrm{pH}$. LAB death, therefore, appeared to be influenced more by bacteriophages rather than $\mathrm{pH}$. Also, Leuconostoc growth was promoted in media supplemented with bacteriophagelysed W. cibaria. Therefore, bacteriophages influenced the life span of LAB during kimchi fermentation. As expected, our results showed that LAB bacteriophages might play an important role in the viability and population dynamics of lactic acid bacteria such as succession involved in kimchi fermentation.

\section{Acknowledgments}

This research was supported by the National Research Foundation of Korea (Grant \# 2017R1D1A1B03033647).

\section{Conflict of Interest}

The authors have no financial conflict of interest to declare.

\section{References}

1. So MH, Kim YB. 1997. Isolation and identification of major microbial groups during Baikkimchi fermentation. Korean J. Food Nutr. 10: 350-359.

2. Kim B, Seo WT, Kim MG, Yun HB, Cho KM. 2012. Metagenomic lactic acid bacterial diversity during Mulkimchi fermentation based on $16 \mathrm{~S}$ rRNA sequence. Appl. Biol. Chem. 55: 787-792.

3. Jeong SH, Jung JY, Lee SH, Jin HM, Jeon CO. 2013. Microbial succession and metabolite changes during fermentation of
Dongchimi, traditional Korean watery kimchi. J. Food Microbiol. 164: 46-53.

4. Jung JY, Lee SH, Jeon CO. 2014. Kimchi microflora: history, current status, and perspectives for industrial kimchi production. Appl. Microbiol. Biotechnol. 98: 2385-2393.

5. Chopin MC, Chopin A, Roux C. 1976. Definition of bacteriophage groups according to their lytic action on mesophilic lactic streptococci. Appl. Environ. Microbiol. 32: 741-746.

6. Kleppen HP, Holo H, Jeon SR, F. Nes I, Yoon SS. 2012. Novel Podoviridae family bacteriophage infecting Weissella cibaria isolated from kimchi. Appl. Environ. Microb. 78: 72997308.

7. Lu Z, Perez-Diaz IM, Hayes JS, Breidt F. 2012. Bacteriophage ecology in commercial cucumber fermentation. Appl. Environ. Microb. 78: 8571-8578.

8. Jung JY, Lee SH, Kim JM, Park MS, Bae JW, Hahn YS, Madsen EL, Jeon CO. 2011. Metagenomic analysis of kimchi, a traditional Korean fermented food. Appl. Environ. Microb. 77: 2264-2274.

9. Lee SH, Jung JY, Jeon CO. 2015. Source tracking and succession of kimchi lactic acid bacteria during fermentation. J. Food. Sci. 80: 1871-1877.

10. Weitz JS, Poisot T, Meyer JR, Flores CO, Valverde S, Sullivan MB, Hochberg ME. 2012. Phage-bacteria infection networks. Trends Microbiol. 21: 82-91.

11. Raya RLR, $\mathrm{H}^{\prime}$ bert EM. Isolation of phage via induction of lysogens. 2009. Bacteriophages: Methods and Protocols, Vol.1: Isolation, Characterization, and Interactions, pp. 2332. Human Press, New York, USA.

12. Wilhelm SW, Suttle CA. 1999. BioScience 49: 781-788.

13. Kang KO, Kim JG, Kim WJ. 1991. Effect of heat treatment and salts addition on Dongchimi fermentation. J. Korean Soc. Food Sci. Nutr. 20: 565-571.

14. Lee MR, Huang YT, Lee PI, Liao CH, Lai CC, Lee LN, et al. 2011. Healthcare-associated bacteraemia caused by Leuconostoc species at a university hospital in Taiwan between 1995 and 2008. J. Hosp. Infect. 78: 45-49.

15. Lee KW, Park JY, Jeong HR, Heo HJ, Han NS, Kim JH. 2012. Probiotic properties of Weissella strains isolated from human faeces. Anaerobe 18: 96-102. 